\title{
Kelix - uma alternativa Linux como base tecnológica para laboratórios educacionais
}

\author{
Marco Antônio Sandini Trentin, Adriano Canabarro Teixeira, Amilton \\ Martins, Marcos José Brusso \\ Curso de Ciência da Computação - Universidade de Passo Fundo (UPF), \\ Caixa Postal 611 - 99010-970 - Passo Fundo - RS - Brasil \\ \{trentin, teixeira, amilton, brusso\} @upf.br
}

Resumo. Esse artigo descreve a experiência do projeto Kit Escola Livre - Kelix, da Universidade de Passo Fundo - UPF, no desenvolvimento de uma solução tecnológica livre para atender a demanda de software educacional e de apoio à formação de cidadãos críticos, produtores de conhecimento e criativos. O trabalho inicia pela fundamentação filosófica do Software Livre como agente na distribuição de acesso ao conhecimento e de liberdade. São abordadas as tecnologias utilizadas e suas formas de contribuição ao objetivo do projeto, bem como a motivação pela escolha e desenvolvimento das ferramentas. Também são abordados os casos de sucesso no uso do Kelix e as perspectivas de trabalhos futuros.

Palavras-chave: informática educativa, inclusão digital, software-livre.

\section{Kelix - a Linux alternative as technology base for educational laboratories}

Abstract. This article describes the experience of the Kelix - Kit Escola Livre Projetct of the Universidade de Passo Fundo - UPF, in the development of a free technological solution to take care of the demand of educational software and support to the formation of critical citizens, producing of knowledge and creative. The work initiates for the philosophical recital of Free Software as agent in the distribution of access to the knowledge and freedom. The used technologies and its forms of contribution to the objective of the project are boarded, as well as the motivation for the choice and development of the tools. Also are are boarded the cases of success in the use of the Kelix and the perspectives of future works.

Keywords: informatics in education, digital inclusion, free software.

\section{Cenário em composição}

Embora relacionar o acesso dos indivíduos às tecnologias de rede - TR como elemento determinante de inclusão digital seja incorrer em incoerência e simplificação analítica, é fato que ter oportunidade de interação com e através das TR é uma condição fundamental de inclusão social na sociedade contemporânea.

Tal demanda se faz ainda mais forte ao se analisar a situação brasileira, onde, segundo dados da Internet World Stats, aproximadamente $23 \%$ da população brasileira têm acesso à internet (2008). Neste sentido, é fundamental que se proponham alternativas viáveis para a implementação de espaços de acesso às TR, baseados na liberdade, na colaboração e na criatividade. 


\subsection{Inclusão Digital sob um novo paradigma}

Projetos de inclusão digital têm recebido espaço crescente na mídia, algumas vezes em uma perspectiva simplista e superficial acerca da sua real missão. São comuns iniciativas em que parte da população acaba sendo submetida a atividades que remetem à reprodução e à dependência tecnológica, cujo principal objetivo é a formação de mãode-obra barata, direcionada ao domínio de determinadas ferramentas.

Com a popularização dos meios de comunicação e acesso facilitado a tecnologias de rede, geralmente entende-se que incluir digitalmente consiste no fornecimento de acesso às tecnologias, normalmente através de telecentros, ou ainda, através do fornecimento de computadores a classes menos favorecidas financeiramente, sem qualquer preocupação ou avaliação do real impacto social que essa tecnologia poderá reproduzir no cotidiano destes indivíduos, ou qualquer tipo de acompanhamento para uma apropriação crítica e criativa desses recursos.

Este processo de disponibilização de acesso é realidade nas mais diversas áreas da atividade humana, entretanto, na Educação em especial, reveste-se de importância na medida em que mais do que acesso, é preciso que os processos educativos se apropriem destas tecnologias em uma dinâmica de qualificação da aprendizagem.

É de fundamental importância orientar e difundir a inclusão digital calcada sob a visão da busca pela construção de uma Sociedade da Aprendizagem, formada por cidadãos críticos e livres, capazes de serem agentes ou nós construtores de conhecimento nesta grande rede. O princípio básico da formação desses indivíduos deve estar ligado ao estímulo constante à produção intelectual e à autonomia, onde cada um se vê naturalmente como participante e autor no processo de disseminação e produção de conhecimento, respeitando suas características físicas, intelectuais e culturais, e, principalmente em uma dinâmica de respeito à diversidade.

\subsection{Projetos universitários a serviço da comunidade}

Baseados nesses conceitos e buscando, além do compartilhamento do acesso, a autonomia e (re)integração de grupos excluídos à essa Sociedade da Aprendizagem tão distante, o projeto filantrópico Mutirão pela Inclusão Digital ${ }^{1}$ da Universidade de Passo Fundo tem trabalhado com grande êxito. Os grupos atendidos encontram muito mais que tecnologia e comunicação na medida em que participam de atividades de resgate à auto-estima e de exercício da cidadania. Entre os atendidos pelas oficinas estão crianças de escolas públicas de periferia, jovens infratores e pessoas com necessidades especiais.

A visão de ligação intrínseca entre a Inclusão Digital e a Cidadania é muito discutida e já faz parte do plano de ação do Governo Brasileiro e do Instituto de Tecnologia da Informação, ao denunciarem que "a exclusão digital amplia a miséria e dificulta o desenvolvimento humano local e nacional". Ainda,

"a inclusão digital não pode ser apartada da inclusão autônoma dos grupos sociais pauperizados, ou seja, da defesa de processos que assegurem a construção de suas identidades no ciberespaço, da ampliação do multiculturalismo e da diversidade a partir da criação de conteúdos próprios na Internet, e, pelo ato de cada vez mais assumir as novas tecnologias da informação e comunicação para ampliar sua cidadania" (Silveira, 2008).

Entretanto, Teixeira (2005) denuncia que "uma parcela da sociedade ainda imersa em uma utilização passiva das tecnologias contemporâneas às utiliza em uma perspectiva linear, verticalizada e hierarquizada, em uma dinâmica de passividade

1 Projeto de Extensão Comunitária da UPF - Disponível em http://www.inf.upf.br/ mutirao V. 6 № 1 , Julho, 2008 
e recepção, garantindo desta forma a manutenção da organização social contemporânea essencialmente fundada no consumo e na reprodução".

Neste sentido, diferente de muitas iniciativas que buscam o treinamento em tecnologias digitais, que replicam conceitos e encapsulam tecnologias para que sejam aceitas sem questionamento, as ações do Mutirão buscam o resgate do raciocínio, da criatividade e a proposta de atividades que propiciem a construção de conhecimento, evitando a transposição de velhos conceitos sob uma roupagem tecnológica.

\subsection{Software Livre como ferramenta de inclusão digital}

Durante as oficinas do mutirão, constatou-se a necessidade de uma linhagem de softwares voltados para a aprendizagem, com ferramentas "livres e pela liberdade". A opção de uso de Software Livre era óbvia, pois garante a convergência entre a filosofia do projeto e dos seus meios. Podemos apontar que "desvincular Software Livre e sua filosofia de ações de Inclusão Digital, representa, além da incoerência teórico-conceitual uma ação contrária à opção nacional potencialmente orientada à criação de uma cultura de colaboração, comunicação, exercício da cidadania e democratização do conhecimento" (Teixeira, 2005).

A partir da experiência e organização do Mutirão, foram selecionadas algumas opções funcionalmente viáveis para apoio de software. Dentre as mais variadas distribuições GNU/Linux ${ }^{2}$ com esse objetivo, como o Edubuntu $^{3}$, o Freeduc ${ }^{4}$ e o Skolelinux $^{5}$, o grupo optou pelo projeto Kdeedu ${ }^{6}$ e seus aplicativos. A idéia não era buscar um ambiente infantilizado, e sim um ambiente com proximidade visual dos sistemas já conhecidos, expandindo-o com aplicativos direcionados aos objetivos do projeto.

\section{Kelix - O nascimento uma solução tecnológica livre}

O projeto Kit Escola Livre - Kelix $^{7}$ foi concebido inicialmente para instrumentalizar as ações do projeto Mutirão pela Inclusão Digital. Desta forma, iniciou-se em 2005 a concepção e desenvolvimento de um conjunto de ferramentas e uma coletânea de softwares educacionais. Surgiu então o Kelix, como uma distribuição Linux para iniciativas de inclusão digital e uso em laboratórios de escolas e telecentros. Optou-se pela utilização de softwares não-proprietários, para uma dimensão de apropriação da filosofia colaborativa, libertadora e inclusiva que fundamenta o Software Livre.

Após a definição das necessidades do grupo, foi feito um trabalho de seleção e avaliação de softwares do Kdeedu, dentre outras fontes, buscando aplicativos que pudessem auxiliar no cumprimento dos objetivos, primando pela interatividade, criatividade, experimentação e autonomia dos usuários. Uma lista inicial de 25 aplicativos foi avaliada, sendo em pouco tempo complementada com ajuda de alunos e professores pesquisadores do Curso de Ciência da Computação.

Entre suas características técnicas, o Kelix permite três formas de operação: (1) fornece subsídios em software para montagem de telecentros ou laboratórios de escolas

2 Mais conhecida ferramenta que utiliza o Software Livre como base filosófica. Sites disponíveis em http://www.fsf.org e http://www.gnu.org

3 Disponível em http://www.edubuntu.org

4 Disponível em http://sourceforge.net/projects/ofset

5 Site oficial disponível em http://www.skolelinux.org/pt_BR/

6 Site oficial disponível em http://edu.kde.org

7 Disponível em http://kelix.upf.br

V. 6 № 1 , Julho, 2008 
usando a tecnologia LTSP para reutilização de computadores antigos; (2) possibilita a instalação em rede com computadores novos e servidor para compartilhar Internet; (3) autoriza a instalação monousuário.

$\mathrm{Na}$ sua versão atual (2.0), o Kelix consiste em um conjunto de pacotes disponibilizados para instalações baseadas no padrão Kubuntu Linux, de forma a auxiliar na implantação, configuração e utilização dos laboratórios de informática. Além da base de operação para o laboratório, o Kelix possui um painel de Software Livre Educacional (Figura 1), contendo mais de 30 aplicativos para uso pelo Ensino Fundamental, prevalecendo softwares de apoio a descoberta e autonomia dos usuários.

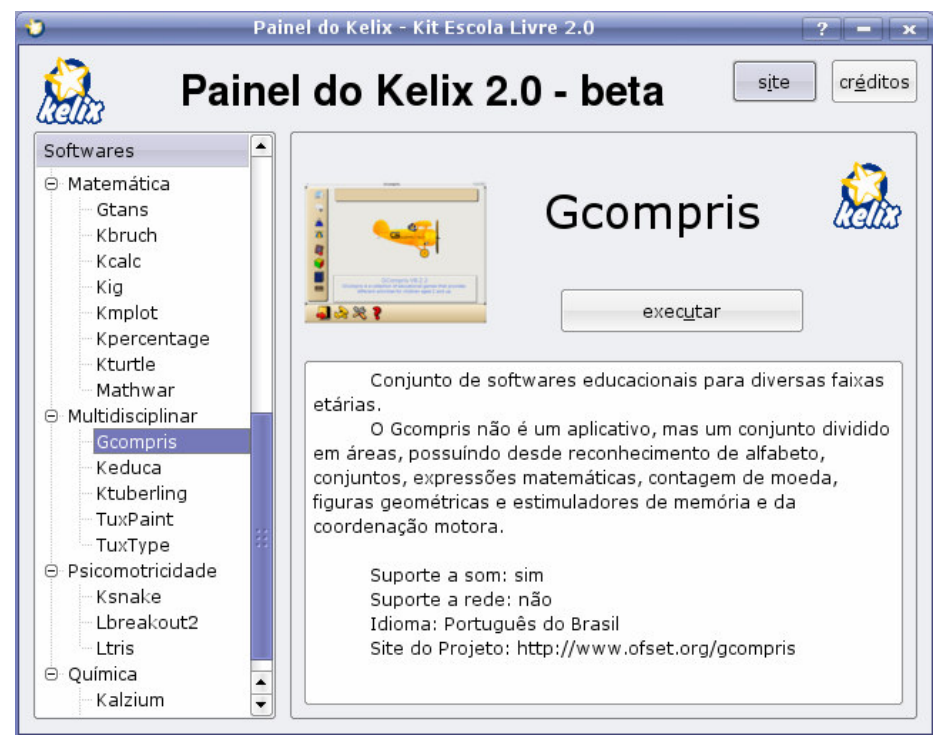

Figura 1 - Painel de acesso aos softwares do Kelix.

Com o objetivo de facilitar o acesso aos softwares selecionados e ao gerenciamento de contas de usuários, foram construídos painéis para instalação, acesso, atualização e configuração dos softwares educacionais, do sistema operacional e da administração de usuários. Por exemplo, na Figura 2, pode-se visualizar um painel de uso por professores e laboratoristas responsáveis pela administração de um laboratório, automatizando e facilitando as tarefas administrativas mais usuais.

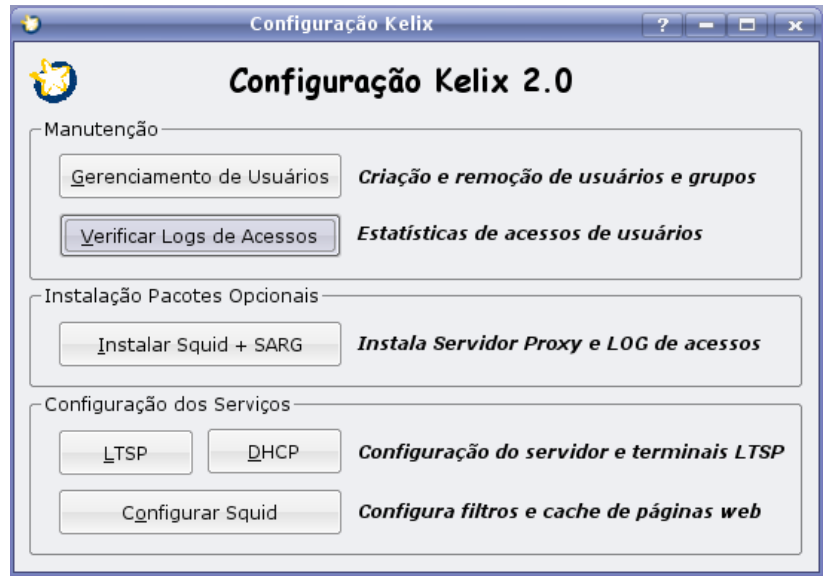

Figura 2 - Painel de administração do Kelix. 
A figura 3, por sua vez, apresenta o painel que automatiza funções de atualização de pacotes e instalação de software com potencial educacional, especialmente os relacionados à web e à manipulação de elementos multimídia.

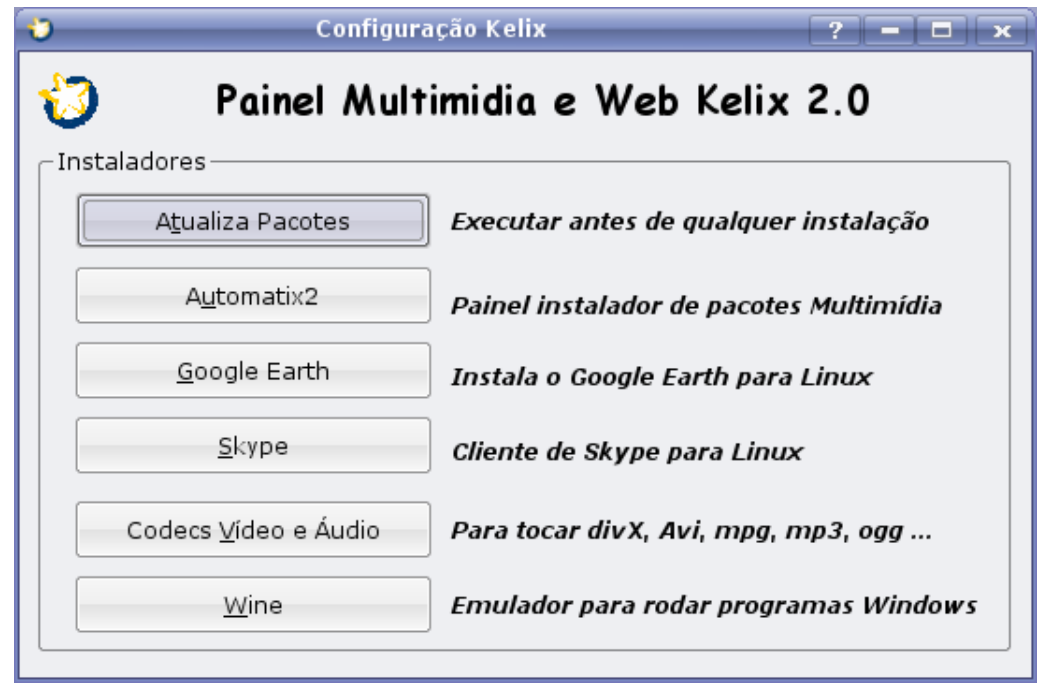

Figura 3 - Painel multimídia e web do Kelix.

Quando os painéis estavam tomando forma, o projeto ganhou o nome de Kit Escola Livre, e posteriormente o codinome KELIX. A palavra Kit vem do "ready to use", empacotado, pronto para usar sem fazer muitas mudanças. O público alvo são pessoas com pouco conhecimento técnico. O termo "Escola Livre" por sua vez aponta para o foco para o qual foi desenvolvido, escolas ou instituições de ensino, mas não no sentido "escola 4 paredes", e sim escola comunitária, participativa, aberta e livre, fundamentada no Software Livre, e tudo que ele representa de participação, cooperação e união. A idéia está centrada na distribuição de conhecimentos, fazendo um contraponto com a filosofia do software livre.

Entretanto, é importante ressaltar que o Kelix consiste em uma solução que vai além da coletânea de softwares. O projeto como um todo prevê, além deste conjunto de softwares, assim como outras distribuições já o fazem, a criação de espaços integrados de disponibilização de softwares educacionais, acessível a todos os usuários, e um espaço colaborativo de socialização de experiências didático-pedagógicas realizadas nas escolas com apoio do Kit Escola Livre.

Portanto, chama-se atenção para o fato de que o Kelix procura atender três frentes diferentes: 1) disponibilizar uma coletânea categorizada de softwares educacionais; 2) manter um repositório dinâmico de softwares educacionais continuamente alimentado por seus usuários; e 3) oportunizar um espaço de socialização de experiências, principalmente por professores usuários do Kelix, a partir de um wiki.

\subsection{Softwares de apoio à Educação}

Após a garimpagem e avaliação de vários softwares livres de cunho educacional e compatíveis com os objetivos do projeto, os mesmos foram catalogados e incluídos na versão empacotada e disponibilizada no site do projeto. A versão 2.0 conta com mais de 
30 softwares de uso educacional. Abaixo são apresentadas as principais categorias e respectivos softwares existentes atualmente:

- Língua Portuguesa: Kdict, Khangman, Klettres, Ktouch;

- Lógica: Codebreaker, Eboard Frozen-Bubble Kbattleship, Kblackbox, Lmemory, Kenolaba , Kreversi, Ksimus, Ksokoban, Pingus;

- Matemática: Gtans, Kbruch, Kcalc, Kig, Kmplot, Kpercentage, Kturtle, Mathwar;

- Multidisciplinar: Gcompris, Keduca, Ktuberling, TuxPaint, TuxType;

- Psicometricidade: Ksnake, Lbreakout2, Ltris;

- Química: Kalzium ,Katomic;

- Geografia: Kstars, Kworldclock;

- Língua Estrangeira: Kverbos.

Todos esses aplicativos são distribuídos em licenças de software livre e estão contidos na versão completa, sem necessidade de downloads extras na Internet.

\section{Usando o Kelix como solução para laboratórios educacionais}

Um recurso bastante interessante incluído no Kelix é o Linux Terminal Server Project $^{8}$. A idéia principal desse projeto é o uso de ThinClients ${ }^{9}$, que são formas inteligentes e funcionais de reaproveitamento de parques de computadores antigos. Com essa tecnologia, é possível agregar dezenas de microcomputadores, mesmo aqueles praticamente obsoletos nos dias atuais, funcionando como terminais, conectados a um servidor que realiza todo o processamento e armazenamento centralizado. Esse servidor, por sua vez, deve possuir recursos atualizados para suprir toda uma rede, inclusive com cotas mínimas de memória $\mathrm{RAM}^{10}$ para cada terminal que ele irá servir, disco rígido de tamanho suficiente para todos os usuários e aplicativos.

Utilizando um protocolo leve, o LTSP usa os terminais apenas como dispositivos de entrada e saída, não necessitando conter disco rígido, drives de disquete ou CD-ROM (Filitto, 2007). Existem equipamentos com tamanho e consumo reduzidos, desenvolvidos especificamente para esses protocolos, suportando diversos sistemas operacionais e servidores de terminais diferentes.

Essa tecnologia atende com muita eficácia a necessidade de suprir a falta de recursos existentes em laboratórios comunitários e escolares. Embora se reconheça que se tem avançado nesta área, a realidade brasileira, ainda é de pouco recurso para investimento em equipamentos de informática, e quase nenhum para manutenção dos mesmos. Muitos dos laboratórios instalados hoje são doações da iniciativa privada, universidades e projetos de inclusão digital por todo país, sendo em geral computadores com poder computacional ultrapassado.

A partir da possibilidade de suprir tecnologicamente uma lacuna econômicosocial tão profunda, a equipe de desenvolvimento do Kelix buscou aliar seus aplicativos a um padrão de configuração testado e pronto para instalar, construindo assim laboratórios usando LSTP. Com isso foram instalados laboratórios de informática em escolas e telecentros de inclusão digital, aproveitando recursos e disponibilidade de equipamentos de projetos de doação de microcomputadores.

8 Site oficial disponível em http://www.ltsp.org

9 Equipamentos de reduzido poder de processamento e armazenamento. Informações disponíveis em http://www.thinclients.net

10 Para um bom desempenho, sugere-se 256MB de RAM acrescida de $128 \mathrm{MB}$ por terminal.

V. 6 № 1 , Julho, 2008 


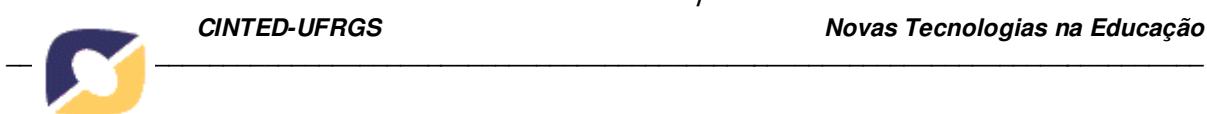

\subsection{Requisitos técnicos}

De acordo com as especificações do mantenedor do LTSP, a necessidade de hardware para o servidor que será o agente tecnológico principal do laboratório está centrada principalmente em disponibilidade de memória RAM para atender a alocação dos aplicativos.

Nos testes feitos pela equipe do Kelix no seu espaço modelo de telecentro com 10 terminais leves, foi encontrada uma configuração mínima de servidor para a aceitável execução dos aplicativos, sendo:

- Processador $2.8 \mathrm{GHz}$;

- Disco rígido Serial Ata $80 \mathrm{~GB}$;

- Placa mãe offboard com suporte a DualChannel;

- Memória RAM 1GB.

Além dessa configuração básica é desejável ter gravador de CD/DVD, placa de rede Gigabit Ethernet, processador dual core e discos rígidos adicionais para cópia espelhada como opcionais.

Já nos terminais de acesso, a configuração mínima é bem mais modesta, limitando apenas a uso de placas de rede $100 \mathrm{Mbps}$ e placas de vídeo com suporte a resolução 1024X768 para uma apropriada execução dos programas gráficos. Em testes executados foi possível executar aplicativos em terminais com processador de $100 \mathrm{MHz}$ e com 32MB de RAM, sem disco rígido.

\subsection{Boot Remoto e soluções em rede}

As soluções de rede devem ser bem projetadas, pois o tráfego de dados pela rede é alto, uma vez que todo armazenamento e execução são feitos pelo servidor. Uma das principais exigências é a rede trafegar a $100 \mathrm{Mbps}$, a fim de evitar o gargalo na comunicação, que causará atraso na execução dos aplicativos, tanto nas placas de rede dos terminais, quanto na do servidor. Outra opção a ser feita para se obter um bom desempenho de todos os terminais é o uso de um Switch com uma porta Gigabit Ethernet, e nela conectar o servidor.

Para a inicialização dos terminais pela rede é necessário o uso de um software de boot remoto, que irá carregar o kernel em memória, montar o sistema de arquivos remotamente usando NFS (inclusive o swap), inicializar o servidor X e finalmente carregar o terminal gráfico (Filitto, 2007).

Para essa função pode ser utilizado o software PXE11, que é compatível com diversas placas de rede existente no mercado e pode ser encontrado em várias mídias, podendo ser disquetes de boot, CDs de boot ou uma versão minimalista que pode ser gravada em EPROMs de $128 \mathrm{~KB}$, podendo com isso ficar conectada direto na placa de rede, simplificando o processo de inicialização, mas acabando por encarecer os terminais, uma vez que essas memórias ROM não são fáceis de encontrar prontas e sua gravação exige equipamento específico. Atualmente, a maioria das placas de rede onboard possui suporte nativo a PXE, facilitando em muito a inicialização dos terminais.

11 Versões em várias mídias disponíveis em http://www.rom-o-matic.com V. 6 № 1, Julho, 2008 


\section{Desdobramentos da experiência: o Kelix na comunidade}

No final de 2005 iniciou-se uma parceria entre a Universidade de Passo Fundo, através do Curso de Ciência da Computação e a Secretaria Municipal de Educação de Passo Fundo com vistas à implantação de laboratórios de informática em dez escolas municipais. Todos os laboratórios foram instalados com a distribuição do Kelix. Em paralelo ao processo de implantação dos laboratórios, iniciou-se a discussão acerca da importância da capacitação de professores das escolas que seriam contempladas com esses laboratórios.

Em novembro de 2006, iniciou-se um curso de 180 horas, para 60 professores da rede municipal de ensino, composto por cinco módulos, com vistas à fomentar uma apropriação criativa das tecnologias. Os módulos previam, além da utilização do Kelix, das ferramentas da Internet e do Pacote BrOffice, a Construção de Projetos de Aprendizagem que pudessem explorar as possibilidades que um laboratório de informática pode propiciar na escola (Trentin, 2007). Outras capacitações semelhantes a essa continuam acontecendo com professores de escolas municipais, bem como oficinas diversas relacionadas ao Kelix em eventos do curso, tais como nos Seminários de Inclusão Digital e Software Livre, que realizou em 2008 sua $3^{\text {a }}$ edição.

Desde a implantação dos primeiros laboratórios nas escolas municipais, a UPF vem dando suporte na instalação e configuração e atualização do Kelix. Atualmente 14 escolas da rede municipal de ensino de Passo Fundo possuem laboratórios de informática com o Kelix, sendo que cada laboratório possui 1 servidor e 10 terminais. Uma vez que dentro da política de informatização das escolas municipais, cada laboratório deve assumir o papel de telecentro, fornecendo acesso à toda a comunidade do entorno da escola, estima-se que o Kelix tem sido usado por mais de 20.000 cidadãos passofundenses.

Está previsto ainda para esse ano a implantação de mais 16 laboratórios, possibilitando assim que todas as escolas da rede municipal de ensino disponham de laboratórios de informática. Todos possuem acesso a Internet e utilizam o Kelix. O Kelix também é utilizado em laboratórios em escolas e telecentros de outras cidades próximas, tais como Soledade, Getúlio Vargas, Lagoa dos Três Cantos, entre outras.

\section{Horizontes a serem trilhados}

O Kelix está fundamentado em distribuir não somente uma mídia, mas na prestação de serviços de apoio, técnico inclusive, para instalação de telecentros, na formação dos docentes e laboratoristas, na criação de um site dinâmico que possa disponibilizar ferramentas de comunicação para a troca de experiências entre seus usuários, softwares, área download e avaliação e tutoriais. No LiveCD encontram-se softwares de cunho educacional ou entretenimento, uma vez que se acredita que os dois dissociados não produzem tanto efeito quanto juntos, o Linux Terminal Server pronto para instalar, e ainda material de apoio aos laboratoristas.

Buscamos com esse trabalho uma alternativa de ferramenta de combate ao apartheid digital, sendo que está em constante avaliação e melhoria, participando ativamente como base de apoio tecnológico às atividades do Mutirão pela Inclusão Digital. A iniciativa do grupo mantenedor do Kelix é a de romper as fronteiras limitadas V. 6 № 1, Julho, 2008 


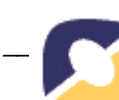

pelo uso exclusivo do LiveCD, buscando fornecer uma série de serviços de apoio ao uso e melhoramento do Kelix.

Entre esses serviços, será desenvolvido um portal de inclusão digital, contendo material de estudos on-line, grupos de apoio e parceiros nas ações de inclusão, estudos de caso, central de iniciativas livres educacionais e atualizações de software. Este espaço tem dois objetivos. $\mathrm{O}$ primeiro é fornecer apoio às comunidades geograficamente distantes da nossa área de atuação, que muitas vezes têm muitas dificuldades de iniciar ou conduzir projetos dessa natureza. E, o segundo, consiste no suporte ao desenvolvimento de projetos interdisciplinares que se constituam em efetivo processo de aprendizagem com características reticulares, colaborativas e cooperativas, envolvendo as escolas municipais de Passo Fundo.

Além do suporte tecnológico, pretendemos constituir uma equipe interdisciplinar de professores das áreas atendidas pelo Kelix, que faça constantes avaliações, traduções e sugira melhorias aos autores dos softwares nele contidos. Essa equipe deverá conter também técnicos que suprirão a necessidade de instalação, treinamento e suporte aos laboratórios onde o Kelix estará presente.

Como todo tipo de iniciativa livre, construída por voluntários e pesquisadores, muitas são os desafios para a execução de nosso trabalho, porém ainda maiores são as convicções de que é urgente a construção de alternativas livre e baseadas na colaboração e na partilha, especialmente quando se trata de qualificar processos educativos.

\section{Referências}

FILITTO, D. OLIVEIRA, J. A relevância da Tecnologia LTSP na inclusão Digital. Saber Acadêmico - Revista Multidisciplinar da Uniesp. São Paulo, n. 4, dezembro de 2007.

INTERNET WORLD STATS: Usage and Population Statistics. Disponível em: <http://www.internetworldstats.com/stats15.htm>. Acesso em: 26 mai. 2008.

SILVEIRA, Sérgio Amadeu. - "Inclusão Digital, Software Livre e Globalização ContraHegemônica". Disponível em: <http://www.softwarelivre.gov.br/artigos/>. Acesso em: 10 mai. 2008.

TEIXEIRA, Adriano Canabarro. "A indissociabilidade entre Inclusão Digital e Software Livre na Sociedade Contemporânea: a experiência do Mutirão pela Inclusão Digital”. Tese de doutorado, (2005).

TRENTIN, M. ; TEIXEIRA, A. C. ; BERTOLETTI, A. C. ; ROSSETO, M. C. (2007). "Formação docente: um exercício de autonomia colaborativa". RENOTE. Revista Novas Tecnologias na Educação, v. 1, p. 1. 